\title{
Low frequency radio monitoring of Cygnus X-1 and Cygnus X-3
}

\author{
M. Pandey ${ }^{1}$, A. P. Rao ${ }^{2}$, G. G. Pooley ${ }^{3}$, P. Durouchoux ${ }^{4}$, R. K. Manchanda ${ }^{5}$, and C. H. Ishwara-Chandra ${ }^{2}$ \\ 1 Dept. of Physics, Mumbai University, Mumbai 400 098, India \\ e-mail: mamta@ncra.tifr.res.in \\ 2 NCRA, TIFR, Post Bag 3, Ganeshkhind, Pune 411 007, India \\ 3 Cavendish Laboratory, University of Cambridge, Madingley Road, Cambridge CB3 OHE, UK \\ ${ }^{4}$ CNRS FRE 2591/CEA Saclay, DSM/DAPNIA/SAP, 91191 Gif-sur-Yvette Cedex, France \\ 5 Tata Institute of Fundamental Research, Mumbai 400005, India
}

Received 16 May 2005 / Accepted 4 October 2005

\begin{abstract}
We present the results of monitoring observations of the micro-quasars Cygnus X-1 and Cygnus X-3 at 0.61 and $1.28 \mathrm{GHz}$. The observations were performed with the Giant Meter-wave Radio Telescope between 2003 June and 2005 January. Variable, unresolved sources were found in both cases. Cyg X-1 was detected in about half of the observations, with a median flux density about $7 \mathrm{mJy}$ at each frequency. The variations in Cyg X-1 may be due to refractive interstellar scintillation. Cyg X-3 was detected in each observation, and varied by a factor of 4 . For this source, models of the scintillation suggest a very long timescale (the order of $40 \mathrm{yr}$ at $1.28 \mathrm{GHz}$ ), therefore, we believe that the variations are intrinsic to the source.
\end{abstract}

Key words. binaries: general - ISM: jets and outflows - radio continuum: stars - accretion, accretion disks - radiation mechanisms: general

\section{Introduction}

The X-ray sources Cygnus X-1 and Cygnus X-3 are accreting X-ray binary systems that have been classified as microquasars. The hard X-ray emission in such systems is believed to originate in the inverse Comptonization of seed photons in the accretion disc; the sources exhibit several spectral states classified as low-hard, intermediate, and high-soft state (Cui 1999). Spectral analysis of the radio emission from these X-ray binaries with black hole candidates (BHCs) suggests that the radio emission arises due to synchrotron emission from the high energy electrons emitted in the jets (Hjellming \& Johnston 1988; Hjellming \& Han 1995) and that the radio emission is quenched when a source is in the high-soft X-ray state (Corbel et al. 2000), and when it is detected in both low-hard and intermediate X-ray state. High-resolution mapping of X-ray binaries, particularly the well-known object GRS $1915+105$, has demonstrated the presence of relativistic outflows (hence the term "micro-quasars"). Such motions have been mapped in Cyg X-3 (Mioduszewski et al. 2001), and a jet-like feature has been mapped in Cyg X-1 (Stirling et al. 2001). There has been a variety of other studies, mostly at high radio frequencies (above $2 \mathrm{GHz}$ ). However, the low frequency characteristics of Cyg X-1, in particular, have not been explored so far.

To understand the physical mechanism connecting the inflow and outflow of matter in such systems, a comprehensive study of the correlation between radio and X-ray emission in different states of these sources is necessary. We monitored micro-quasars systematically using the Giant Meter-wave Radio Telescope, GMRT (Pandey et al. 2004), at low frequencies. In this paper, we present the observations of Cyg X-1 and Cyg X-3, which demonstrate variations in the flux densities at low radio frequencies for both these sources. The observed flux density variations can be ascribed either to intrinsic mechanisms specific to each source, i.e. a process occurring around the compact sources themselves, or to refractive interstellar scintillation. The refractive scintillation can be described as the flux density variations caused by "focusing" and "defocusing" of electromagnetic waves by large-scale, plasma density irregularities in the interstellar medium (Rickett et al. 1984).

\section{Observations and analysis}

The radio snapshot observations (of duration $30 \mathrm{~min}$ ) were carried out at $0.61 \mathrm{GHz}$ and $1.28 \mathrm{GHz}$ with a bandwidth of 16/32 MHz using the Giant Meter-wave Radio Telescope, GMRT. The flux density scale was set by observing the primary calibrators $3 \mathrm{C} 286$ and $3 \mathrm{C} 48$. Phase calibrators were interleaved with 25-min scans on each of Cyg X-1 and Cyg X-3. The sampling time was $16 \mathrm{~s}$. The data recorded from GMRT was converted into FITS files and analyzed using the Astronomical 
Table 1. Flux density of Cyg X-1 between Jun., 2003 and Jan., 2005.

\begin{tabular}{|c|c|c|c|c|c|c|c|c|}
\hline \multirow{2}{*}{$\begin{array}{c}\text { Date } \\
\text { MJD } \\
v / \mathrm{GHz}\end{array}$} & \multicolumn{2}{|c|}{$\begin{array}{c}S_{v} \\
(\mathrm{mJy})\end{array}$} & \multirow[t]{2}{*}{$\begin{array}{l}\text { GMRT rms } \\
\text { noise (mJy) }\end{array}$} & \multicolumn{2}{|c|}{$\begin{array}{c}\text { Control Source } \\
(\mathrm{mJy})\end{array}$} & \multirow{2}{*}{$\begin{array}{l}S_{v} \\
(\mathrm{mJy}) \\
15\end{array}$} & \multirow[t]{2}{*}{$\alpha$} & \multirow[t]{2}{*}{$\begin{array}{l}\text { X-ray } \\
\text { state }\end{array}$} \\
\hline & 0.6 & 1.2 & & 0.6 & 1.2 & & & \\
\hline 52796 & - & $\leq 4.8$ & 1.6 & - & 4.2 & 13 & $\geq 0.6$ & $\mathrm{H}-\mathrm{S}$ \\
\hline 52799 & - & 10.1 & 1.3 & - & 5.2 & 22 & 0.3 & H-S \\
\hline 52827 & $\leq 2$ & - & 0.5 & 9.1 & - & 8 & $\geq 0.4$ & $\mathrm{H}-\mathrm{S}$ \\
\hline 52829 & $\leq 1.5$ & - & 0.4 & 8.9 & - & 13 & $\geq 0.5$ & $\mathrm{H}-\mathrm{S}$ \\
\hline 52845 & - & $\leq 7$ & 2.2 & - & 4.7 & 6 & $\geq 0.06$ & $\mathrm{H}-\mathrm{S}$ \\
\hline 52848 & - & $\leq 5$ & 1.3 & - & 4.8 & 6 & $\geq 0.07$ & $\mathrm{H}-\mathrm{S}$ \\
\hline 52855 & - & 5.4 & 0.2 & - & 5.7 & 22 & 0.8 & Int \\
\hline 52891 & 5.8 & - & 0.7 & 9.2 & - & 15 & 0.3 & L-H \\
\hline 53037 & - & 9.2 & 0.2 & - & 4.4 & 15 & 0.2 & Int \\
\hline 53102 & 9.7 & - & 0.4 & 10.3 & - & 22 & 0.3 & L-H \\
\hline 53104 & $\leq 7$ & - & 2.0 & 8.9 & - & 27 & $\geq 0.4$ & L-H \\
\hline 53107 & 10.3 & - & 0.7 & 10.3 & - & 26 & 0.3 & L-H \\
\hline 53127 & $\leq 7$ & - & 2.1 & 10.5 & - & 16 & $\geq 0.3$ & L-H \\
\hline 53377 & $\leq 5.1$ & - & 1.7 & 8.7 & - & 6 & $\geq 0.05$ & H-S \\
\hline 53379 & $\leq 3.3$ & - & 1.1 & 8.5 & - & 6 & $\geq 0.2$ & H-S \\
\hline 53391 & 8.4 & - & 0.9 & 9.5 & - & 20 & 0.3 & Int \\
\hline 53392 & 8.4 & - & 0.8 & 9.3 & - & 16 & 0.2 & Int \\
\hline
\end{tabular}

Image Processing System (AIPS). A self-calibration on the data was used to correct for phase-related errors and improve the image quality. Tables 1 and 2 summarize details of observations using GMRT at the two frequencies between June 2003 and Jan. 2005. Cyg X-3 was detected in each observation, with Cyg X-1 during about one-half of them. Columns 1-3 in both the tables give the dates of observations and the measured flux densities or upper limits. Column 4 gives the corresponding rms noise in the field of the radio image. For the observations performed during telescope maintenance/test time, the background noise was higher because of the reduced number of antennas available. For each field, we detected about 6 background sources. We record the measured flux density of one from each field in Tables 1 and 2, at an angular distance of 15' for Cyg X-1 and 22' for Cyg X-3. The measured flux densities of these "control" sources are consistent with constant actual flux densities and the observed noise levels. This result gives us overall confidence in the reliability of the system.

To supplement the data from GMRT, we also used radio data on Cyg X-1 and Cyg X-3 taken with the Ryle Telescope at $15 \mathrm{GHz}$. The longitudes of the GMRT and Ryle Telescope differ by $74 \mathrm{deg}(5 \mathrm{~h})$, so the observations are usually not exactly simultaneous; in the worst cases the nearest $15-\mathrm{GHz}$ observation is separated by 2 days from the GMRT observation. The radio light curve at $15 \mathrm{GHz}$ for $\mathrm{Cyg} \mathrm{X}-1$ was averaged for $10 \mathrm{~min}$, that for $\mathrm{Cyg} \mathrm{X}-3$ for $5 \mathrm{~min}$. The typical uncertainty is $2 \mathrm{mJy}+3 \%$ in the flux scaling. In Col. 6 of the tables we list the flux density at $15 \mathrm{GHz}$ for the nearest observation at that frequency. Column 7 gives the spectral indices between the GMRT observation and $15 \mathrm{GHz}$ (in the sense $S \propto v^{\alpha}$ ). Column 8 gives the X-ray spectral state of the source as determined from the 2-12 keV light curves of the sources taken from the ASM archival data.

In Figs. 1 and 2 we show the first ever radio images of Cyg X-1 and Cyg X-3 at 0.61 GHz. The AIPS subroutine jmfit shows that the images are consistent with point sources.

\section{Discussion}

\subsection{Cyg $X-1$}

Cyg X-1 is bright X-ray source with mass in excess of $7 M_{\odot}$ (Gies \& Bolton 1986) and a distance of $\sim 2.5 \mathrm{kpc}$. The companion is an O9.7 Iab super-giant (V1357 Cyg, HDE 226868), showing a strong stellar wind (Persi et al. 1980) and almost filling its Roche lobe (Bolton 1972). The observed radial velocity for the system $v \sin i$ is about $76 \mathrm{~km} \mathrm{~s}^{-1}$ (Gies $\&$ Bolton 1982). The emission in X-rays and in the radio band vary over a wide range of time-scales, including modulation at the 5.6 orbital period; see, for example, Brocksopp et al. (1999) and Gleissner et al. (2003). The X-ray emission is usually in the spectral state described as "low-hard" (with a non-thermal component extending to a few hundred $\mathrm{keV}$ ); the relatively rare "high-soft" state is dominated by a strong lowenergy component. The high-frequency radio emission tends to be suppressed during the high-soft states (Hjellming et al. 1975) (see Fig. 4). While the X-ray source is in the low hard state, the radio emission in the cm-wave band is persistent, but the magnitude does vary by a factor of about 5, with very rare outbursts to higher flux densities. These variations are much less spectacular than those of some other members of this class, such as Cyg X-3 and GRS 1915+105. The radio spectral index $\alpha$, defined in the sense $S \propto v^{\alpha}$, is close to 
Table 2. Flux density of Cyg X-3 between Jun., 2003 and Jan., 2005.

\begin{tabular}{cllllllll}
\hline \hline $\begin{array}{c}\text { Date } \\
\text { MJD }\end{array}$ & \multicolumn{2}{c}{$\begin{array}{c}S_{v} \\
(\mathrm{mJy})\end{array}$} & $\begin{array}{l}\text { GMRT rms } \\
\text { noise }(\mathrm{mJy})\end{array}$ & \multicolumn{2}{c}{$\begin{array}{c}\text { Control Source } \\
(\mathrm{mJy})\end{array}$} & $\begin{array}{l}S_{v} \\
(\mathrm{mJy})\end{array}$ & $\alpha$ & $\begin{array}{l}\text { X-ray } \\
\text { state }\end{array}$ \\
$v / \mathrm{GHz}$ & 0.6 & 1.2 & & 0.6 & 1.2 & 15 & & \\
\hline 52797 & - & 57.9 & 0.4 & - & 7.7 & 70 & 0.08 & L-H \\
52800 & - & 90.4 & 0.5 & - & 9.6 & 110 & 0.08 & L-H \\
52830 & 31.2 & - & 0.8 & 16.6 & - & 115 & 0.4 & L-H \\
52849 & - & 62.4 & 0.4 & - & 9.4 & 190 & 0.5 & L-H \\
52856 & - & 53.5 & 0.3 & - & 8.4 & 132 & 0.4 & L-H \\
52892 & 7.0 & - & 0.7 & 17.8 & - & 73 & 0.7 & L-H \\
53037 & - & 21.2 & 0.3 & - & 8.1 & 51 & 0.4 & L-H \\
53105 & 14.5 & - & 1.1 & 16.6 & - & 45 & 0.4 & L-H \\
53107 & 13.0 & - & 0.8 & 20.1 & - & 70 & 0.5 & L-H \\
53127 & 40.8 & - & 1.1 & 18.6 & - & 50 & 0.06 & L-H \\
53128 & 29.5 & - & 0.8 & 19.5 & - & 73 & 0.3 & L-H \\
53378 & 31.4 & - & 1.7 & 18.7 & - & 130 & 0.4 & H-S \\
53379 & 29.8 & - & 1.1 & 18.5 & - & 130 & 0.5 & H-S \\
53392 & 27.3 & - & 0.9 & 19.5 & - & 170 & 0.6 & H-S \\
53393 & 44.2 & - & 0.8 & 19.3 & - & 170 & 0.4 & H-S \\
\hline
\end{tabular}

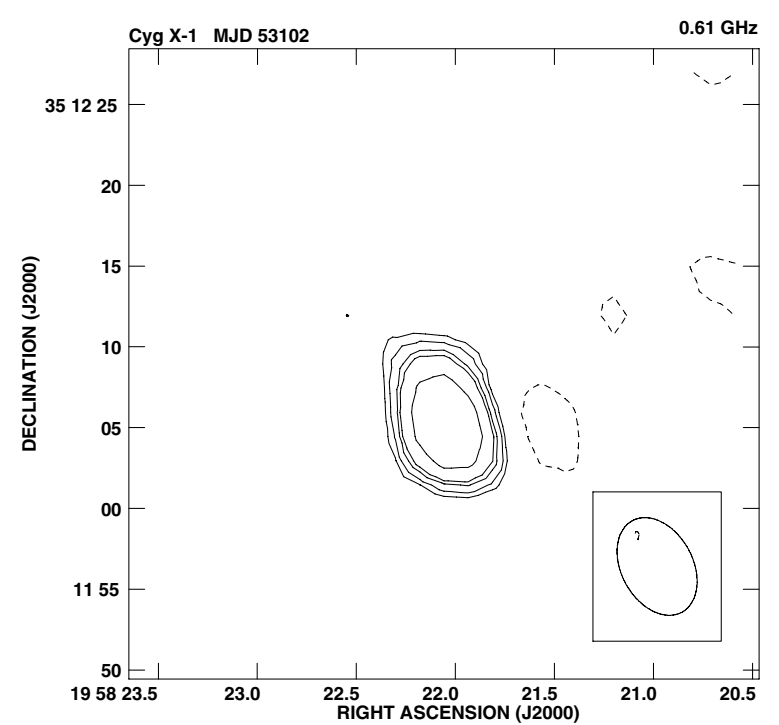

Fig. 1. GMRT image of CygX-1. The contour levels are $1.3 \mathrm{mJy} /$ beam $\times(-1,1,1.4,2,4,8)$.

zero in the range 2-220 GHz (Fender et al. 2000). Jet emission in Cyg X-1 has also been observed at milliarcsec resolution during the low-hard state at $8.4 \mathrm{GHz}$ (Stirling et al. 2001). This jet is also not as dramatic (in terms of rapid variability and relativistic outflows) as in the cases of jets observed in other microquasars, e.g. Cyg X-3 (Miouduszeski et al. 2001), GRS 1915+105 (Dhawan et al. 2000), IE 1740.7-2942 (Mirabel et al. 1992), and GRS 1758-258 (Rodríguez et al. 1992). Observations by Gallo et al. (2005), however, suggest that the jet may be inflating a large $(5 \mathrm{pc})$ shell-like structure around the X-ray source, implying that its contribution to the outflow of energy in this system is dominant. Figure 3 shows

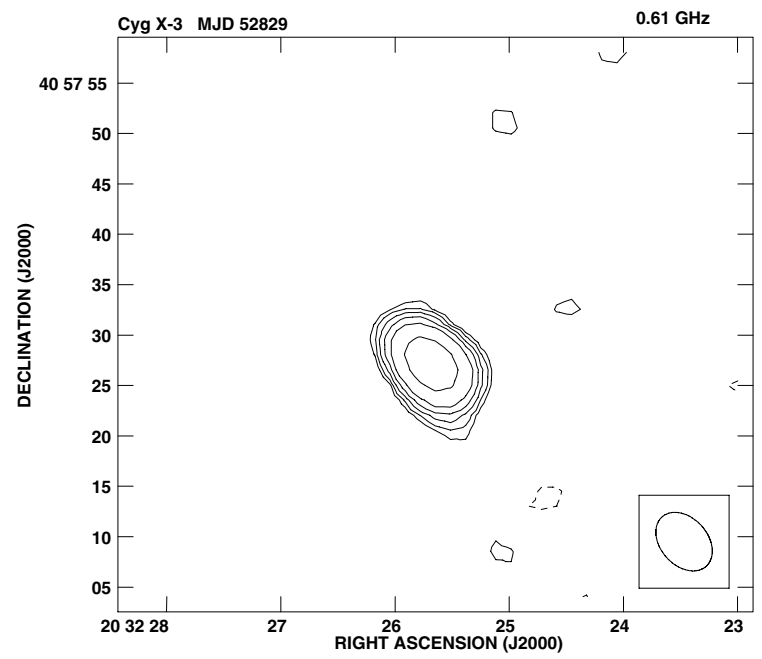

Fig. 2. GMRT image of CygX-3. The contour levels are $2.3 \mathrm{mJy} /$ beam $\times(-1,1,1.4,2,4,8)$.

the low-frequency flux density from GMRT plotted against the closest available 15-GHz flux density. Different markers are used for the two GMRT frequencies. There is a clear correlation between the high- and low-frequency data, which somewhat complicated by the upper limits on some of the GMRT flux densities, but is nevertheless convincing.

\subsubsection{The radio and X-ray association of Cyg X-1}

The $15-\mathrm{GHz}$ radio light curve and the RXTE/ASM X-ray count-rate and hardness ratio $\mathrm{HR} 2$ for $\mathrm{CygX}-1$ from Mar., 2003 to Jan., 2005 are shown in Fig. 4, where daily averages for both of the X-ray parameters are used. The X-ray state of the system changed several times over this interval. 


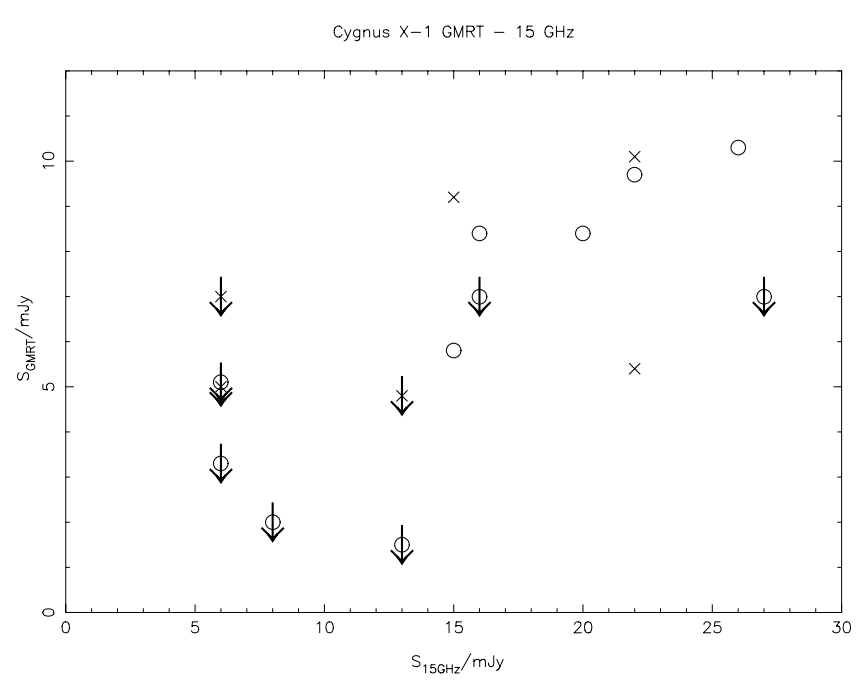

Fig. 3. Flux-flux plot for Cyg X-1 showing the low-frequency GMRT flux densities $(\bigcirc 0.61 \mathrm{GHz}, \times 1.28 \mathrm{GHz})$ and the closest 15-GHz measurement.

For the majority of the time, Cyg X-1 was in the low-hard state, but with three distinct periods of the high-soft state lasting 1-2 months each. The suppression of the 15-GHz flux density during the high-soft states is illustrated well by these data.

There was one exceptional flare recorded in the $15 \mathrm{GHz}$ data, on Feb. 20, 27, 2004 where the flux density reached a maximum of $140 \mathrm{mJy}$ in an event that lasted about $30 \mathrm{~min}$. Unfortunately, there was no GMRT observation on this date.

\subsubsection{Spectral and temporal behavior of Cyg X-1}

Cyg X-1 is a persistent but variable flat-spectrum source over the range 2 to $220 \mathrm{GHz}$ (Fender et al. 2000). It is clear from Table 1 and Fig. 3 that Cyg X-1 was detected at low frequency in only one of the 8 observations, while it was in the highsoft X-ray state. Observations in the intermediate and lowhard X-ray states all resulted in detections, apart from two that both had relatively high noise levels. Therefore we conclude that the high-soft state suppresses the radio emission at low radio frequencies, as it does at high frequencies. There is no clear distinction between the radio spectral index of the intermediate and low-hard states, typically $\alpha=0.3$. While all of these spectral measurements are subject to additional uncertainty from the lack of precisely simultaneous observations, we also conclude that the radio spectrum rises from the GMRT observing frequencies to the regime where it is essentially flat ( 2 to $220 \mathrm{GHz}$ ). Part of the variation of radio emission from Cyg X-1 is associated with the X-ray state of the system, and is therefore intrinsic. A further part may be associated with propagation through the interstellar medium; in particular, refractive interstellar scintillation (RISS) may introduce amplitude variations (Rickett 1990). We used the NE2001 code made available by Cordes \& Lazio (1993) to estimate the propagation parameters for Cyg X-1 and Cyg X-3.

The NE2001 code estimates that the transition frequency (above which the scattering is weak, and therefore the intensity fluctuations small) is around $6 \mathrm{GHz}$. The angular broadening is estimated as 0.3 mas at $0.61 \mathrm{GHz}$ and 0.06 mas at $1.28 \mathrm{GHz}$. The scattering disc is therefore about $1 \times 10^{11} \mathrm{~m}$ at $0.61 \mathrm{GHz}$ and $2 \times 10^{10} \mathrm{~m}$ at $1.28 \mathrm{GHz}$. If we assume that typical transverse velocities involved (the source or the medium) are near $100 \mathrm{~km} \mathrm{~s}^{-1}$, we derive timescales of 5 and 1 days at the two frequencies. These are clearly in the same area as those of the observed fluctuations, so it is quite possible that RISS is a contributing factor. We note, however, two further points. The angular size of the (transient) radio jet mapped at $8.4 \mathrm{GHz}$ by Stirling et al. (2001) is 10 mas: the low-frequency emission may originate in a region of this size (or larger), and the scintillation bandwidths estimated by the NE2001 code are 30 and $100 \mathrm{MHz}$ at $0.61,1.28 \mathrm{GHz}$. Both of these would reduce the observed RISS contribution to the fluctuations.

\subsubsection{Proposed geometry and emission mechanism}

From the above results, we conclude that the radio spectrum of Cyg X-1 consists of two parts, the persistent high frequency emitting component and the variable low frequency component. Both of these components imply the presence of a continuous jet with discrete plasmoids or clouds of electrons emitting synchrotron photons. The observed behavior of the source at radio frequencies can be explained by adiabatically expanding plasmoids in conical jets (Hjellming et al. 1988), where the low frequency radio emitting component is significantly altered by refractive interstellar scintillation giving rise to high variability in the radio flux density. A sketch of the proposed geometry is shown in Fig. 5. In our model, compact plasmoids are ejected continuously at the base of the jet and expand freely outward. The low frequency emission from the newly ejected plasmoids will be absorbed due to synchrotron self absorption in an optically thick medium because of its highly compact nature and high magnetic field. The plasmoid will still be visible at high radio frequencies (Miller-Jones et al. 2004). As the plasma expands under its internal pressure, it will become optically thin and emit over the whole radio-frequency band, although at a lower intensity.

The observed flat radio spectrum of Cyg X-1 can thus be explained as due to superposition of individual self-absorbed synchrotron components that are emitted or plasmoids with differing age profile and with a longer lifetime. If a combination of size and the magnetic field are such that opacity effects are only important for the younger population, an inverted (or overall flat) spectrum is seen. The average spectrum can also mimic a flux level shift above a cut-off frequency, characterized by the optical thickness of the medium and the choice of age distribution. As discussed earlier, the 15-GHz and GMRT flux densities are correlated; while we do not believe that the $15-\mathrm{GHz}$ data are significantly affected by scintillation, this may not be the case at the GMRT frequencies, and at least some of the variance in the 0.61 and $1.28 \mathrm{GHz}$ data results form RISS. In conclusion, the radio emission in $\mathrm{Cyg} \mathrm{X}-1$ is mainly due to a continuous outflow of either compact plasmoids or electron clouds into a jet, while the spectrum consists of the composite emission from each of these slowly decaying ejecta, and mini flaring events, 


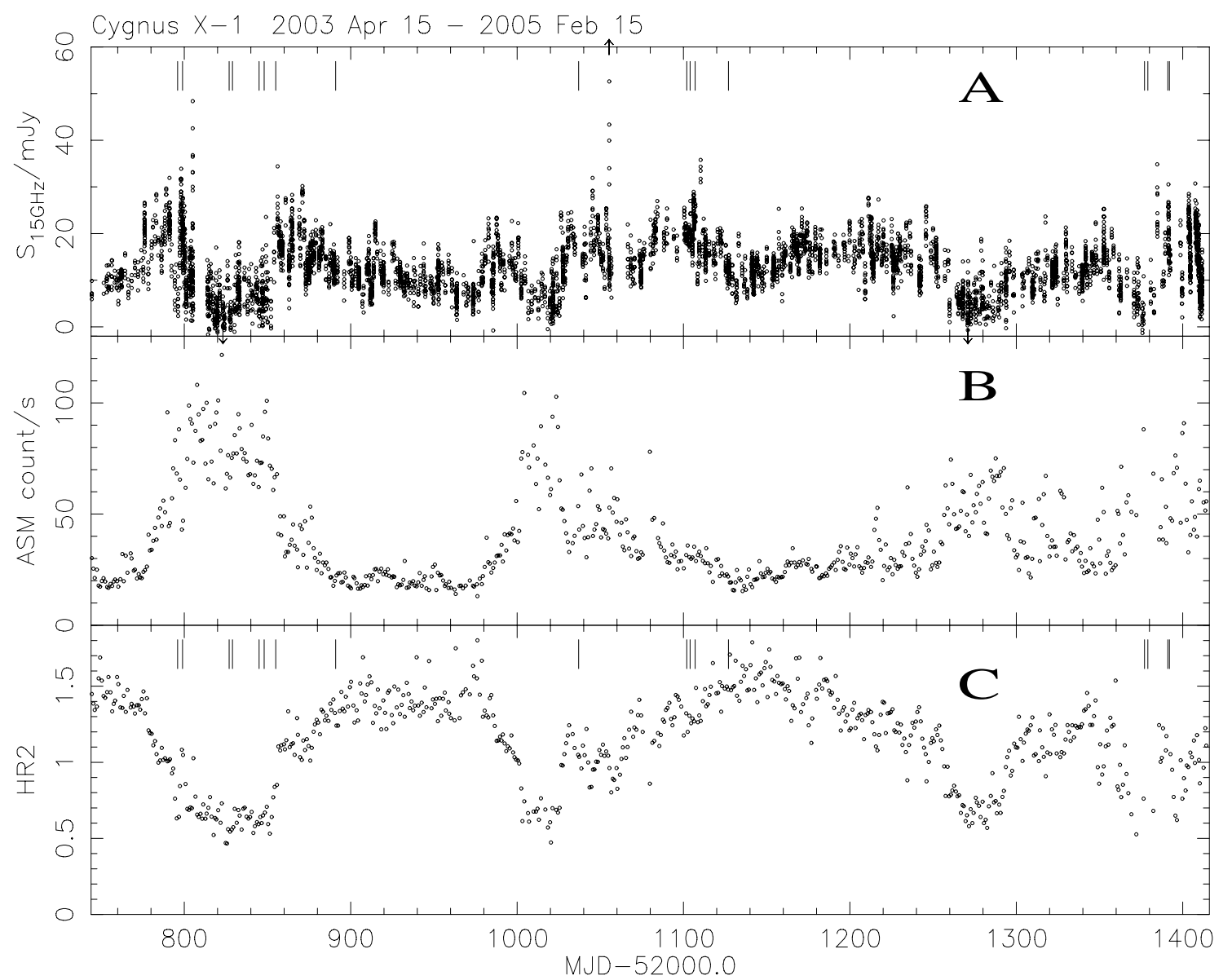

Fig. 4. Cygnus X-1: Radio (15 GHz) (top) and RXTE/ASM (middle) light-curves; ASM hardness ratio HR2 = (5-12) keV/(3-5) keV (bottom) during the interval MJD $52796=2003$ Jun. 6 to MJD $53392=2005$ Jan. 22. The dates of GMRT observations are marked with vertical lines.

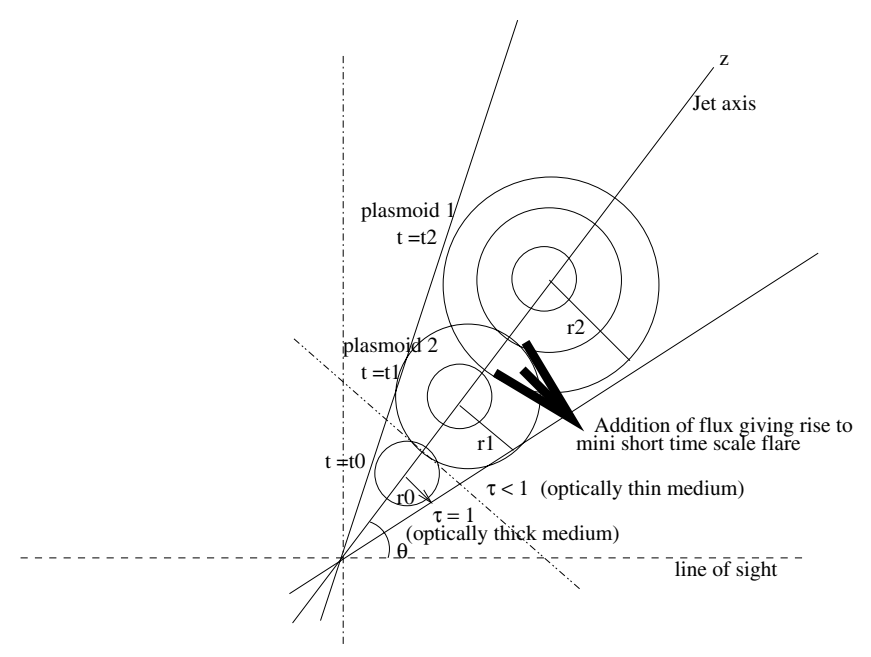

Fig. 5. Model for the Cyg X-1 jet.

as observed in the source, may result due to random age distribution of the ejected clouds.

\section{2. $C y g X-3$}

Cyg X-3 is an X-ray binary system that does not seem to fit easily into the established classes of these systems. It has a 4.8-h orbital period, and is located in the Galactic plane at a distance of $\sim 10 \mathrm{kpc}$ (Predehl et al. 2002; Dickey 1983; Mason et al. 1979). The optical counterpart of the X-ray source is a WR star (van Kerkwijk et al. 1996), that is not visible in the optical band because of heavy interstellar extinction, but is clearly seen above $\sim 0.8 \mu \mathrm{m}$. The infrared data also shows a 4.8 -h modulation corresponding to the orbital period as seen in X-rays (Hanson et al. 1999). The source exhibits two spectral states of low-hard and high-soft similar to Cyg X-1 and has been observed up to $500 \mathrm{keV}$ during the flare mode. The quasi-periodic oscillations with periods between 50-1500 s are the key characteristics of the source (van der Klis \& Janson 1985). Detailed analysis of the X-ray spectrum suggests that the total number of X-ray photons seems to be conserved at all times irrespective of the state, and the observed spectrum is consistent with a thermal source embedded in a hot plasma and enveloped in a cold hydrogen shell (Manchanda 2002). The X-ray light curve of the source in the $2-12 \mathrm{keV}$ band from the RXTE/ASM data shows frequent spectral changes between the high-soft to lowhard states, thereby suggesting large changes in the accretion rate on to the compact object.

At radio wavelengths, Cyg X-3 is the most luminous X-ray binary in both its quiescent and flaring states (Waltman et al. 1995). Huge radio outbursts have been reported in Cyg X-3, during which the flux density can increase up to levels 


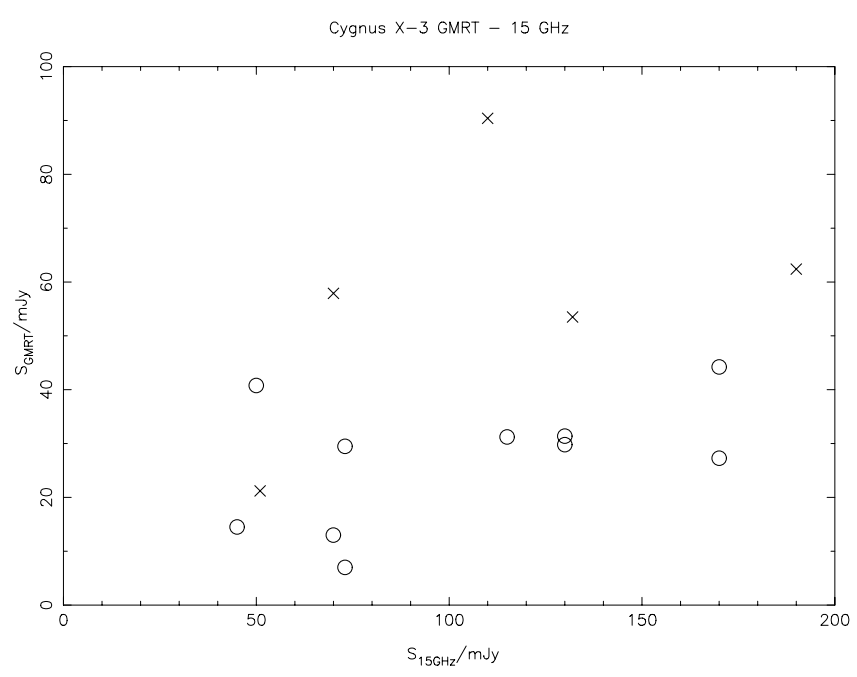

Fig. 6. Flux-flux plot for Cyg X-3 showing the low-frequency GMRT flux densities $(\bigcirc 0.61 \mathrm{GHz}, \times 1.28 \mathrm{GHz})$ and the closest 15-GHz measurement.

of $\sim 20 \mathrm{Jy}$; radio emission is suppressed ("quenched") to levels below $1 \mathrm{mJy}$ for some days before large radio flares (Waltman et al. 1994). Jet-like structures with repeated relativistic ejection have been observed at various radio frequencies (e.g. Schalinski et al. 1998). On an arc-second scale, two-sided jets have been seen from the source in the $\mathrm{N}-\mathrm{S}$ orientation, whereas a highly-relativistic $(\beta \geq 0.81)$, one-sided jet with the same orientation has been reported on milli-arcsec scales with the VLBA (Martí et al. 2001; Mioduszewski et al. 2001).

\subsubsection{Temporal characteristics in the radio band and their association with the X-ray emission}

In Table 2 above we summarize the radio flux densities of the source as measured during various observations with GMRT and from the Ryle Telescope data. Figure 6 shows a flux-flux plot for Cyg X-3 for the GMRT and 15-GHz data, and Fig. 7 shows the $15-\mathrm{GHz}$ and RXTE ASM data for the whole period. The timing of the GMRT observations is again marked with vertical lines in Fig. 7. The last 4 GMRT observations, all at $0.61 \mathrm{GHz}$, were made during the high-soft state. The mean flux density at that frequency was $33.2 \mathrm{mJy}$, compared with $22.7 \mathrm{mJy}$ for the 6 previous data points; the $15-\mathrm{GHz}$ mean value was also higher; $150 \mathrm{mJy}$ compared with $71 \mathrm{mJy}$ for the corresponding 6 data points. We note that the radio/X-ray correlation is the opposite of that for Cyg X-1. McCollough et al. (1999) report both anti-correlations (in the quiescent state) and correlations (in the flaring state) between the hard X-ray flux (20-100 keV, as measured by BATSE) and the $\mathrm{cm}$-wave radio flux density of Cyg X-3.

We investigated the possibility that RISS might be important in the case of Cyg X-3. The propagation conditions are severe, than for the case of Cyg X-1: the path length is much longer, and it has been known for some time (e.g. Wilkinson et al. 1994) that the scatter-broadening for this source is extreme. The NE2001 model is consistent, it suggests angular broadening of 20.59, $4.03 \mathrm{arcsec}$ at $0.61,1.28 \mathrm{GHz}$. The corresponding timescales would be many years, and the narrow scintillation bandwidth $(2 \mathrm{~Hz})$ would suppress any observed scintillation. We conclude that RISS is not relevant to this study of Cyg X-3.

To look for correlation between the radio emission from the source with its X-ray emission characteristics, we plotted the RXTE/ASM X-ray light curve for Cyg X-3 in Fig. 7 along with the radio data. The timing of the GMRT observations is shown by the vertical lines. It can be seen that no large flares were observed during this interval. The radio emission is in the "quiescent" state, typically 50 to $200 \mathrm{mJy}$ at $15 \mathrm{GHz}$. For the time-range 53380-53392, the X-ray spectrum softens: the RXTE ASM ratio HR2 falls consistently below 2 , and the radio emission starts to become more erratic. This behavior faded away after another month or so, and the source returned to the quiescent state.

\subsubsection{Spectral behavior and the emission geometry}

As seen from the data in Table 2 and Fig. 6, Cyg X-3 is a persistent radio source at all wavelengths. Cyg X-3 is more luminous at higher frequencies. The data in Table 2 clearly indicates a low frequency turnover in the source spectrum below $1 \mathrm{GHz}$. As discussed earlier, such behavior can arise due to synchrotron self absorption of the compact radio emitting plasma in an optically thick medium. The observed variability of the flux density is consistent with the assumption of a discrete ejection/plasmoid in adiabatic expansion. The ejection rate and/or lifetimes of plasmoids in Cyg X-3 are probably lower/shorter than in Cyg X-1 with little or no overlap of the spectra associated to the single discrete ejections. The spectrum and the fact that the radio emission is persistently at high level may therefore imply an uniterrupted low-rate ejection.

\section{Summary}

In this paper we have presented the first ever low frequency maps of Cyg X-1 and Cyg X-3, along with the, temporal behavior, after monitoring observations of these sources over two years at various frequencies. It was noted that, while Cyg X-3 is persistent source at low frequencies, Cyg X-1 is transient in nature with a mean radio flux of $7 \mathrm{mJy}$, and the source is detected at low radio frequencies only when the high frequency radio flux crosses the mean threshold of $15 \mathrm{mJy}$. The spectral characteristics of both sources also have many similarities, e.g. the spectral turn over at lower frequencies. Continuous blobs of plasmoids are emitted within the jet medium in both cases. However, the lifetime of the individual blob in the case of Cyg X-1 is larger than in the case of Cyg X-3, hence, a flatness in the radio spectra is seen in the case of Cyg X-1. The positive detection of Cyg X-1 at 0.61 to $1.2 \mathrm{GHz}$ on several occasions, along with the spectral turnover at low frequencies, conclusively supports the synchrotron origin of the radio photons contrary to its ambiguous thermal or non-thermal nature as concluded from earlier measurements (Fender et al. 2000).

Rigorous calculations were performed to look for the effect of refractive interstellar scintillation at low frequency on 


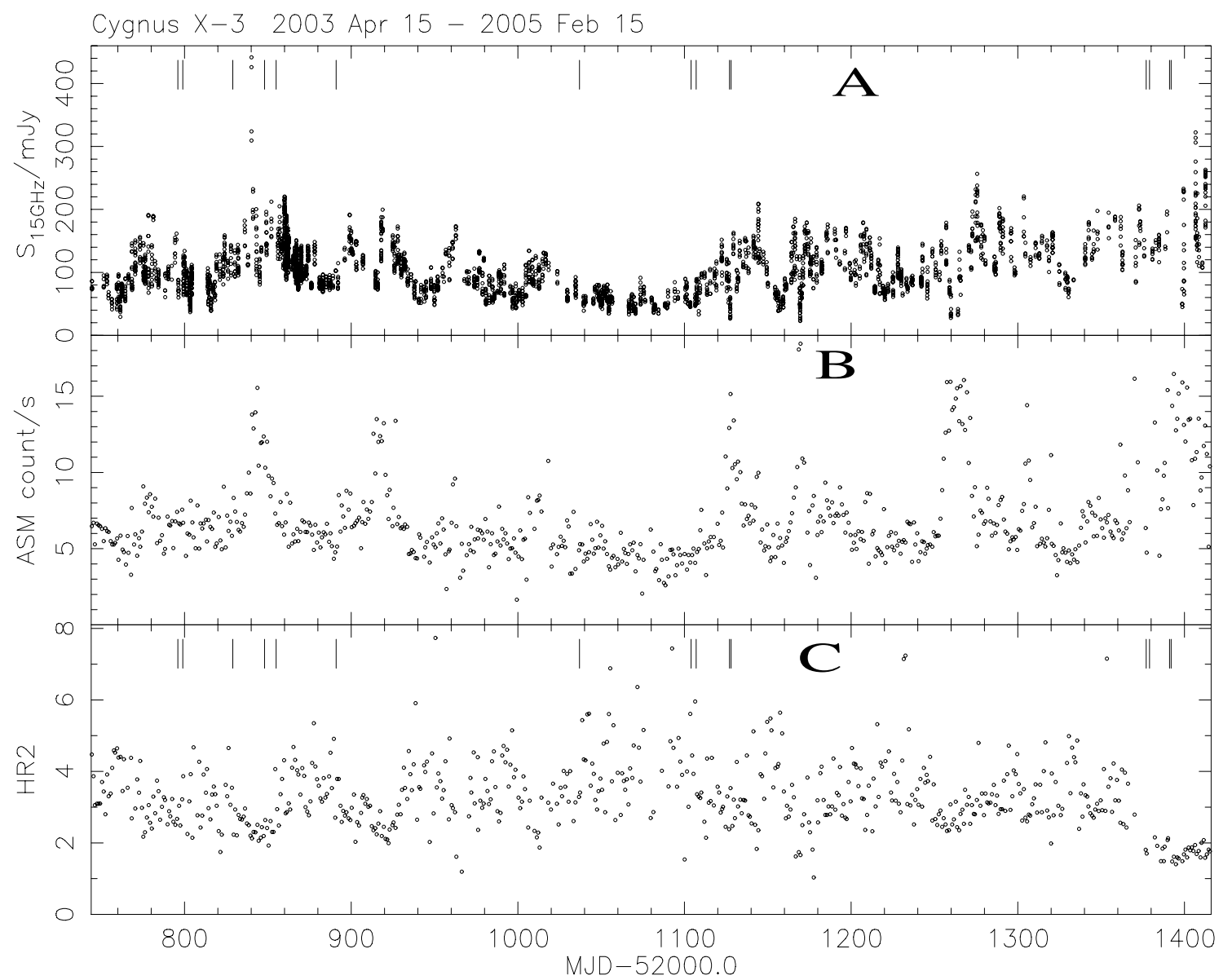

Fig. 7. Cygnus X-3: radio (15 GHz) (top) and RXTE/ASM (middle) light-curves; ASM hardness ratio (bottom), HR2 = (5-12) keV/(3-5) keV during the interval MJD $52796=2003$ Jun. 6 to MJD $53392=2005$ Jan. 22. The dates of GMRT observations are marked with vertical lines.

the data of these two sources. The present data clearly indicate that the source visibility above a threshold luminosity seen in $\mathrm{Cyg}$ X-1 is due to this effect. However, the variability at high frequencies is intrinsic to the source and least affected by interstellar scintillations. Finally, we discussed a common jet emission model for both those sources in which synchrotron radiation-emitting plasmoids originate at the base of the conical jet and the observed spectral features due to the superposition of many such plasmoids with different age profile. Thus detailed spectral measurements in the $\mathrm{m}-\mathrm{cm}$ regime (low frequency radio) combined with simultaneous X-ray data can provide a clear understanding of the emission mechanisms in these sources.

Acknowledgements. We wish to acknowledge the help received from the GMRT staff during observations. MP wishes to thank the NCRA for providing hospitality to carry out this work and acknowledges the financial support received from the, Raman Research Institute Trust, Bangalore, during the early stages of this work. She is also thankful to Prof. V. Kulkarni from Mumbai Univ. for constant encouragement. Part of the work was supported by the Indian Space Research Organization grant under the REPOND program. The RXTE ASM quick-look results provided by the ASM/RXTE team are gratefully acknowledged. The Ryle Telescope is supported by the PPARC.

\section{References}

Bolton, C. 1972, Nature, 240, 124

Braes, L., \& Miley, G. 1971, Nature, 232, 246

Belloni, T., Klein-Wolt, M., Mendez, M., et al. 2000, A\&A, 355, 271

Blandford, R. D. 1989, Theory of accretion Disks, ed. P. Meyer, W. Duschl, J. Frank, \& E. Meyer-Hofmeister (Dordrecht: Kluwer), 35

Brocksopp, C., Fender, R. P., Larionov, V., et al. 1999, MNRAS, 309, 1063

Coles, W. A., Frehlich, R., Rickett, B., et al. 1987, ApJ, 315, 666

Cordes, J. M., Chatterjee, S., \& Lazio, T. 2003, ASPC, 302, 225

Cordes, J. M., \& Lazio, T. 2003, The Cordes-Lazio NE2001 Galactic Free Electron Density Model, http://rsd-www.nrl.navy.mil/7213/lazio/ne_model/

Cordes, J. M., \& Lazio, T. 2004, ASPC, 317, 211

Corbel, S., Fender, R. P., Tzioumis, A. K., et al. 2000, A\&A, 359, 251

Cui, W. 1999, High Energy Processes in Accreting Black Holes, ed. J. Poutanen, \& R. Svensson (San Francisco: ASP), 97

Dhawan, V., Mirabel, I. F., \& Rodríguez, L. F. 2000, ApJ, 543, 373

Dickey, J. 1983, ApJ, 273, L71

Fender, R. P., Pooley, G. G., Durouchoux, P., et al. 2000, MNRAS, 312,853

Gallo, E., Fender, R. P., Kaiser, C., et al. 2005, Nature, 436, 819

Gies, D., \& Bolton, C. 1982, ApJ, 260, 240

Gies, D., \& Bolton, C. 1986, ApJ, 304, 371

Gierlinski, M., Zdziarski, A., et al. 1977, MNRAS, 288, 958 
Gleissner, T., Wilms, J., Pooley, G. G., et al. 2004, A\&A, 425, 1061

Hanson, M. M., Still, M. D., \& Fender, R. P. 1999, A\&AS, 195, 3906

Hjellming, R., Gibson, D., Owen, F., et al. 1975, Nature, 256, 111

Hjellming, R., \& Han, X. 1995, X-ray Binaries, ed. W. H. G. Lewin, J. Van Paradijs, \& E. P. J. van den Heuvel (Cambridge: Cambridge University Press)

Hjellming, R., \& Johnston, J. 1988, ApJ, 328, 600

Ishwar-Chandra, C., Rao, A. P., Pandey, M. D., et al. 2004, Proceedings of 5th Micro-quasar workshop, Chinese Journal of Astro. \& Astrophys

Konigl, A. 1989, ApJ, 342, 208

Konigl, A., \& Ruden, S. P. 1992, Protostars and Planets III, ed. E. H. Levy, \& J. I. Lunine (Univ. of Arizona Press)

Levine, A. M., Cui, W., Remillard, R., et al. 1996, A\&AS, 189, 3511

Mason, K. O., \& Sanford, P. W. 1979, MNRAS, 189, 9

Martí, J., Paredes, J. M., \& Peracaula, M. 2001, A\&A, 375, 476

Manchanda, R. K. 2002, JA\&A, 23, 197

McCollough, M. L., Robinson, C. R., Zang, S. N., et al. 1999, ApJ, 517,951

Miller-Jones, J. C. A., Blundell, K. M., Rupen, M. P., et al. 2004, ApJ, 600,368

Mioduszewski, A. J., Rupen, M. P., Hjellming, R. M., et al. 2001, ApJ, 553,766

Mirabel, I. F., Rodríguez, L. F., Cordier, B., et al. 1992, Nature, 358, 215

Pandey, M. D., Durouchoux, P., Manchanda, R., et al. 2004, Proceedings of 5th Integral workshop, ESA
Persi, P., Ferrari-Toniolo, M., Grasdalen, G. L., et al. 1980, A\&A, 92, 238

Pooley, G., Brocksopp, C., Fender, R. P., et al. 1999, MNRAS, 309, 1063

Predehl, P., Wilms, J., Nowak, M. A., et al. 2002, Proceedings of the 3rd Micro-quasar workshop, ed. A. J. Castro-Tirado, J. Greiner, \& J. M. Paredes (Dordrecht: Kluwer), 41

Rickett, B. J. 1990, ARA\&A, 28, 561

Rybicki, G., \& Lightman, A. 1979, Radiative processes in Astrophysics (New York: Wiley)

Rickett, B. J., Coles, W. A., \& Bourgois, G. 1984, A\&A, 134, 390

Rodríguez, L., Mirabel, F., \& Martí, J. 1992, ApJ, 544, 443

Schalinski, C. J., Johnston, K. J., Witzel, A., et al. 1998, A\&A, 329, 504

Stirling, A., Spencer, R., \& de la Force, C. 2001, MNRAS, 327, 1273

Stinebring, D. R., Smirnova, T. V., Hankins, T., et al. 2000, ApJ, 539

van der Klis, M., \& Janson, F. A. 1985, Nature, 313, 768

van Kerkwjick, M. H., Gebelle, T. R., King, D. L., et al. 1996, A\&A, 314, 521

Waltman, E. B., Fiedler, R. L., Johnston, K. L., et al. 1994, AJ, 108, 179

Waltman, E. B., Ghigo, F. D., \& Johnston, K. J. 1995, AJ, 110, 290

Watanabe, H., Kitamoto, S., Miyamoto, S., et al. 1994, ApJ, 433, 350

Wilkinson, P. N., Narayan, R., \& Spencer, R. E. 1994, MNRAS, 269, 67 